\title{
Neural Substrates Underlying Learning-Related Changes of the Unconditioned Fear Response
}

\author{
Kimberly H. Wood ${ }^{1}$, Dystany Kuykendall ${ }^{1}$, Lawrence W. Ver Hoef ${ }^{2}$ and David C. Knight ${ }^{1, *}$ \\ ${ }^{I}$ Department of Psychology, University of Alabama at Birmingham, Birmingham, AL 35294, UK \\ ${ }^{2}$ University of Alabama at Birmingham, School of Medicine; Birmingham VA Medical Center, UK
}

\begin{abstract}
The ability to predict an impending threat during Pavlovian conditioning diminishes the emotional response that is produced once the threat is encountered. Diminution of the threat response appears to be mediated by somewhat independent associative learning and expectancy-related processes. Therefore, the present study was designed to better understand the neural mechanisms that support associative learning processes, independent of expectancy, that influence the emotional response to a threat. Healthy volunteers took part in a Pavlovian conditioning procedure during which trait anxiety, expectation of the unconditioned stimulus (UCS), skin conductance response (SCR), and functional magnetic resonance imaging (fMRI) signal were assessed. The results showed no evidence for associative learning that was independent of expectation. Threat-related SCR expression was diminished on predictable trials vs. unpredictable trials of the UCS (i.e. conditioned UCR diminution). Similar to SCR, conditioned UCR diminution was observed within the left dorsolateral PFC, dorsomedial PFC, ventromedial PFC, and left anterior insula. In contrast, potentiation of the threatrelated fMRI signal response was observed within left dorsolateral PFC, inferior parietal lobule (IPL), and posterior insula. A negative relationship was observed between UCS expectancy and UCR expression within the dorsomedial PFC, ventromedial PFC, and anterior insula. Finally, the anticipatory fMRI signal responses within the PFC, posterior cingulate, and amygdala showed an inverse relationship with threat-related activation within the brain regions that showed UCR diminution. The current findings suggest that the PFC and amygdala support learning-related processes that impact the magnitude of the emotional response to a threat.
\end{abstract}

Keywords: fMRI, conditioning, unconditioned response, prefrontal cortex, emotion, fear, anxiety, skin conductance.

\section{INTRODUCTION}

Fear is considered an important defense mechanism due to its evolutionary role in survival [1-3]. The ability to form associations between a dangerous event and the cues that predict it allows an organism to better adapt to a changing environment [1,4]. An important aspect of this type of associative learning (i.e. Pavlovian fear conditioning) is that it allows an organism to more effectively avoid, escape, or minimize the impact of an impending threat [3-7]. Thus, from a functional perspective it is the response to the threat itself (i.e. unconditioned stimulus: UCS) that directly impacts survival and therefore may be the most biologically relevant feature of fear learning [4].

During Pavlovian fear conditioning, a neutral conditioned stimulus (CS) is paired with an aversive UCS. The conditioned response (CR) produced by the CS is typically used to index fear expression. Traditionally, CR expression is taken as evidence that an association between the CS and UCS has been formed. In contrast, the unconditioned response (UCR) is generally considered an automatic, unlearned reaction to the aversive UCS. However, prior work has shown that unconditioned skin conductance

*Address correspondence to this author at the CIRC 235H, $15303^{\mathrm{RD}}$ AVE S, Birmingham, AL 35294-0021, Tel: (205) 996-6344;

Fax: (205) 975-6320; E-mail: knightdc@uab.edu responses (SCRs) diminish as associations are formed during conditioning [8-12]. For example, UCR amplitude is decreased to paired vs. unpaired presentations of the CS and UCS $[9,13,14]$. Further, conscious expectation of the UCS has also been shown to diminish UCR expression [10, 1517]. For example, UCR amplitude shows a greater decrease when participants expect a UCS compared to when the UCS is unexpected $[10,15]$. This effect is generally referred to as conditioned UCR diminution.

Prior neuroimaging studies have employed Pavlovian fear conditioning to investigate the neural correlates of conditioned UCR diminution $[10,15,17,18]$. These studies have demonstrated a learning-related decrease in UCR expression within multiple regions of the prefrontal cortex (PFC) [e.g. dorsolateral (dlPFC), dorsomedial (dmPFC), ventromedial (vmPFC)], cingulate cortex [e.g. anterior (ACC), posterior (PCC)], inferior parietal lobule (IPL), anterior insula, and amygdala $[10,15,17,18]$. The findings from this prior work are consistent with other research that suggests the PFC regulates the emotional response [19, 20]. For example, prior work indicates that the PFC projects to the amygdala and provides regulatory control over emotionrelated processes during fear conditioning [1, 19, 21]. Further, the threat-related fMRI signal response within the PFC and amygdala impacts the amplitude of the autonomic response (e.g. SCR) that is produced $[10,15,17,22]$. This 
process appears to be critical for normal, healthy emotional function.

Converging lines of research indicate that healthy emotion regulation relies upon the PFC [19, 20, 23-25], and that anxiety disorders may be linked to insufficient regulatory control from the PFC. Further, PFC dysregulation is associated with increased amygdala reactivity [26-31] and an exaggerated emotional response to threats [11, 32, 33]. For example, prior work has shown that participants with low trait anxiety exhibit greater vmPFC activation compared to participants with high trait anxiety during cued fear conditioning [34]. In contrast, individuals with high trait anxiety showed a diminished vmPFC response that was associated with greater fear conditioned SCRs compared to participants with low trait anxiety [34]. Further, our prior work has demonstrated that unconditioned fMRI signal responses from several brain regions fluctuate with trait anxiety level [17]. Specifically, trait anxiety varied with dlPFC, dmPFC, PCC, and IPL activity such that, as trait anxiety level increased the threat-related fMRI signal response within these brain areas increased [17]. These studies suggest that anxiety level influences the magnitude of anticipatory and threat-related brain activation, which in turn influences the peripheral expression of emotion.

Associative learning and expectancy processes are additional factors that modulate the response produced by a threat. Prior work has demonstrated a reduction in the magnitude of the fMRI signal response once a cue-outcome relationship is established and predictable $[35,36]$. However, when an outcome violates expectations an increase in the magnitude of brain activity is observed $[35,36]$. Further, the magnitude of brain activation in response to an aversive stimulus is dependent upon the expectation of whether the aversive outcome is a certainty or only a possibility $[15,37$, 38]. Prior work has demonstrated that the amplitude of the threat-elicited fMRI signal response within regions of the PFC, insula, cingulate, IPL, and amygdala varies with UCS expectancy $[10,15,38]$. More specifically, as UCS expectancy increases during the CS presentation, the amplitude of the threat response decreases. These findings suggest that conditioned UCR diminution is in part mediated by expectation of the UCS that is supported by regions of the PFC. In turn, these conditioned changes in the brain's response to a threat appear to modify the peripheral emotional response that is expressed. For example, several studies have shown that as UCS expectancy increases the magnitude of unconditioned SCR expression decreases [10, $11,15]$. Further, these findings parallel the conditioned changes observed within the threat-related fMRI signal response $[10,15,17,38]$. Taken together, these findings suggest that conscious expectation of an imminent threat may play an important role in modulation of the emotional response produced. However, prior work has also demonstrated threat-elicited SCRs that did not diminish as expectation increased [17]. For example, unconditioned SCRs produced by a UCS that followed a CS- did not differ from SCRs to a UCS presented alone even though UCS expectancies differed between these conditions [17]. Prior work has also demonstrated greater UCR diminution to a UCS that followed a CS+ compared to a UCS presented after a CS- even when UCS expectancy ratings were equivalent [11]. In addition, diminished unconditioned SCRs have been observed to a UCS that followed a CS+ compared to a UCS that followed a CS- even after participants were informed that the UCS would follow both the CS+ and CS- [12]. Together, these studies suggest that modulation of the threatrelated emotional response is not solely mediated by conscious expectations. Instead, the findings suggest that associative learning processes independent of UCS expectations also influence UCR expression.

Given that prior work suggests UCR diminution is in part mediated by expectancy-independent processes, the present study was designed to better understand associative learning processes that influence UCR expression in the absence of differential UCS expectancies. The aim of this study was to determine the neural substrates that support expectancyindependent conditioned diminution of the emotional response to a threat. Based on prior work, we expected to observe a learning-related decrease in the fMRI signal response produced by the UCS independent of UCS expectancy [11]. Given the importance of the amygdala, dlPFC, dmPFC, and vmPFC in emotion [1, 19, 20, 38], we hypothesized that these brain regions would show UCR diminution during Pavlovian conditioning. Further, we expected threat-related brain activation within these brain regions to vary with individual differences in trait anxiety level. In turn, we expected threat-related amygdala activity to vary with the amplitude of unconditioned SCR expression $[17,22,39-41]$.

\section{MATERIALS AND METHODS}

Participants: Twenty-one healthy right-handed volunteers participated in this study [ 8 male, 13 female; age $=23.05 \pm 0.82$ years (mean \pm SEM); range $=19-34$ years $]$. All participants were included in the UCS expectancy and fMRI data analyses. However, four non-responsive (SCR < $0.05 \mu$ Siemens) participants were excluded from SCR data analyses. Thus, a total of seventeen participants were included in the SCR analyses (7 male, 10 female; age = $23.59 \pm 0.95$ years; range $=19-34$ years). Fifteen participants were included in the secondary fMRI data analyses that examined the relationship between behavior and brain activation ( 6 male, 9 female; age $=23.87 \pm 1.06$ years; range $=19-34$ years). The six participants excluded from these secondary fMRI analyses consisted of the four participants with non-responsive SCR and two participants that did not complete the trait anxiety assessment. All participants provided written informed consent.

State-Trait Anxiety Inventory: Prior to the conditioning session, volunteers completed the State-Trait Anxiety Inventory (STAI; Form Y) for Adults [42]. The STAI is a self-assessment questionnaire that measures state and trait anxiety in terms of general negative affect [43]. The state scale reflects anxiety level at the current moment, whereas the trait scale reflects anxiety level experienced in general [42].

Stimuli: The conditioned and unconditioned stimuli were presented through MR-compatible pneumatic headphones. Two tones (1025 and $1050 \mathrm{~Hz} ; 10 \mathrm{~s}$ duration; $20 \mathrm{~s} \mathrm{ITI})$ that were difficult to discriminate served as the CSs. Our pilot work indicated that stimuli presented at these frequencies can be differentiated when presented back-to-back, but are difficult to discriminate when separated by a 20 s ITI. A loud 


\section{Acquisition Phase}
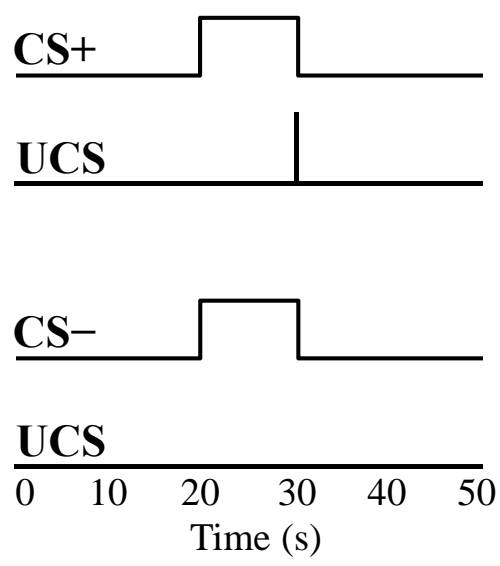

\section{Test Phase}

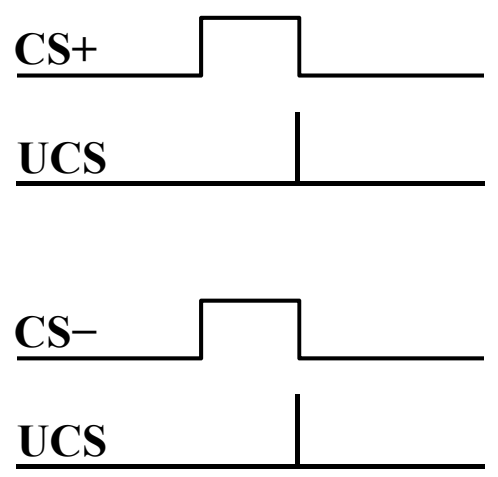

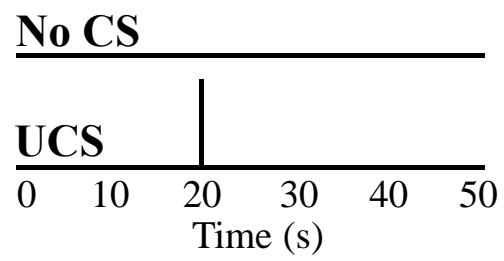

Fig. (1). Conditioned and unconditioned stimuli. Each acquisition scan consisted of CS+ (8 trials), CS- (8 trials), and test trials (1 CS+UCS, $1 \mathrm{CS}-\mathrm{UCS}$, and $1 \mathrm{UCS}$ alone trial). The acquisition phase consisted of four acquisition scans, followed by the test phase. The test phase consisted of $10 \mathrm{CS}+\mathrm{UCS}$ trials, $10 \mathrm{CS}-\mathrm{UCS}$ trials, and $10 \mathrm{UCS}$ alone trials. Stimuli were counterbalanced and presented in a pseudorandom order such that no more than two trials of the same stimulus were consecutively presented.

white-noise served as the UCS (100 db, $0.5 \mathrm{~s}$ duration). Stimuli were presented in a pseudorandom order with the restriction that no more than two trials of the same stimulus could be presented consecutively.

Procedure: Volunteers were exposed to a differential fear conditioning procedure in which the UCS coterminated with one tone $(\mathrm{CS}+)$ and the second tone was presented alone (CS-) during the acquisition phase. The CSs were counterbalanced across participants. The acquisition phase consisted of four $590 \mathrm{~s}$ scans. A total of 32 trials of each CS were presented during the acquisition phase ( 8 trials of each CS were presented in each scan). In addition, each acquisition scan included 3 test trials that consisted of UCS presentations that coterminated with the $\mathrm{CS}+(\mathrm{CS}+\mathrm{UCS})$ and CS- (CS-UCS), as well as presentations of the UCS alone (Fig. 1). Additional details on trial order during the acquisition phase have been published previously [17]. The acquisition phase was followed by a $920 \mathrm{~s}$ test phase that consisted of 30 test trials (10 CS+UCS trials, $10 \mathrm{CS}-\mathrm{UCS}$ trials, $10 \mathrm{UCS}$ alone trials). In total, there were 14 test trials for each stimulus ( 4 from the acquisition phase, 10 from the test phase). The 14 test trials were grouped into the first seven test trials (Early test trials) and the last seven test trials (Late test trials) for further analysis. The test trials were binned in this manner to evaluate learning-related changes in UCS expectancy in a manner consistent with our prior work using CS presentations that were easy to discriminate [17].

UCS expectancy: UCS expectancy was collected throughout the conditioning session. This measure was used to assess participants' expectation of the UCS and determine whether the relationship between the CS and UCS had been learned. Presentation software (Neurobehavioral Systems,
Inc.; Albany, CA), was used to display a UCS expectancy rating scale on an IFIS-SA LCD (Invivo Corp.; Gainesville, FL) video screen. The video screen was located above the participant's head and viewed through a mirror attached to the RF coil. Participant's used an MRI compatible joystick (Current Designs; Philidelphia, PA) to control the rating bar on the video screen. Subjects were instructed to rate their UCS expectancy on a moment-by-moment basis using a continuous scale from 0 to $100(0=$ certain the UCS would not be presented, $50=$ uncertain whether the UCS would be presented, $100=$ certain the UCS would be presented) to reflect their current UCS expectancy. UCS expectancy was calculated as the average response (1 s sample) at UCS onset. Additional details on this methodology have been previously published [44].

Skin conductance response: An MRI compatible physiological monitoring system (Biopac Systems; Goleta, CA) was used to collect SCR data as described in prior work [44]. SCR was sampled $(2,000 \mathrm{~Hz})$ with a pair of disposable radio-translucent electrodes $(1 \mathrm{~cm}$ diameter, Biopac Systems; Goleta, CA) from the distal phalanx of the middle and ring fingers of the nondominant hand. SCR data were processed using Biopac AcqKnowledge 4.1 software. A $1 \mathrm{~Hz}$ low pass digital filter was applied and SCR data were resampled at $250 \mathrm{~Hz}$. Unconditioned SCRs were limited to those that occurred within $10 \mathrm{~s}$ following the UCS presentation. Unconditioned SCRs smaller than 0.05 $\mu$ Siemens were scored as 0 .

Functional MRI: Neuroimaging was completed on a Siemens 3 Tesla Allegra MRI system. High-resolution anatomical images (MPRAGE) were acquired in the sagittal plane using a $\mathrm{T} 1$ weighted series $(\mathrm{TR}=2300 \mathrm{~ms}, \mathrm{TE}=3.9 \mathrm{~ms}$, 
flip angle $=12^{\circ}, \mathrm{FOV}=25.6 \mathrm{~cm}$, matrix $=256 \times 256$, slice thickness $=1 \mathrm{~mm}, 0.5 \mathrm{~mm}$ gap). Whole brain blood oxygen level dependent [45] fMRI was completed using a gradientecho echoplanar pulse sequence [46] in an oblique-axial orientation $\left(\mathrm{TR}=2000 \mathrm{~ms}, \mathrm{TE}=30 \mathrm{~ms}\right.$, flip angle $=70^{\circ}$, $\mathrm{FOV}=24 \mathrm{~cm}$, matrix $=64 \times 64$, slice thickness $=4 \mathrm{~mm}$, no gap) during stimulus presentations. The Analysis of Functional NeuroImages (AFNI) software package [47] was used for the fMRI analyses. Echo-planar time series data were slice time corrected, motion corrected, concatenated, reregistered to the fifth volume of the first imaging block, and spatially blurred using a $4 \mathrm{~mm}$ full-width-at-half-maximum Gaussian filter.

Functional MRI data were analyzed using a deconvolution analysis that was conducted at the individual subject level. This multiple linear regression modeled the input from all stimuli using a gamma variate hemodynamic response function. Reference waveforms modeled the $\mathrm{CS}+$, $\mathrm{CS}^{-}$, and UCS during the acquisition phase, the $\mathrm{CS}+$ and CS- on test trials, joystick movement, and head motion parameters. The regressors of interest modeled brain activation elicited by the UCS during the test trials (i.e. $\mathrm{CS}+\mathrm{UCS}, \mathrm{CS}-\mathrm{UCS}$, and the UCS alone). In this analysis, separate reference waveforms were used for the Early (trials 1-7) and Late (trials 8-14) test trials. Functional MRI percent signal change to the UCS on test trials was used as an index of the amplitude of the neural response to threat. These functional maps were then converted to the Talairach and Tournoux stereotaxic [48] space for group level analyses [49].

Based on prior work $[10,15,17]$, we applied an anatomical mask that consisted of the PFC, cingulate cortex, IPL, insula, and amygdala to reduce the number of voxelwise comparisons for our group level analyses. A repeatedmeasures ANOVA was conducted to test for a main effect of stimulus (CS+UCS, CS-UCS, and UCS alone) and trial (Early vs. Late), as well as a stimulus $\mathrm{x}$ trial interaction. A voxel-wise threshold of $p<0.05$ (corrected) was employed by using an uncorrected threshold of $p<0.005$ and a cluster volume larger than $510 \mathrm{~mm}^{3}$ (9 voxels of $3.75 \times 3.75 \times 4.00$ $\mathrm{mm}$ dimension). Monte Carlo simulations, conducted in AFNI, were used to select these threshold criteria and reject smaller clusters of activation due to chance alone (false positives) [50, 51]. Follow-up t-test comparisons were conducted in SPSS on the mean percent signal change in threat-related brain activation that passed the significance threshold criteria ( $p<0.05$ Bonferroni corrected) revealed by the ANOVA.

Two different analysis procedures (i.e. correlation and multiple linear regression) were conducted to investigate the relationship between threat-elicited brain activation and behavior (i.e. trait anxiety, UCS expectancy, and unconditioned SCR expression). These analyses were restricted to the brain regions that demonstrated learningrelated changes from the ANOVA (i.e. functional regions of interest; ROI). Although these analyses are similar there are important differences (see [17] for additional discussion). In short, separate correlation analyses assessed the relationship between each of our behavioral measures and the mean percent signal change within an ROI as a whole, while the regression analysis evaluated these relationships on a voxelwise basis. The four participants without measurable SCR data and the two participants without a trait anxiety score were excluded from this regression analysis because there were no data points to include in the model. AlphaSim [47, 51] was used to conduct Monte Carlo simulations limited to the functional ROI revealed by our repeated-measures ANOVA that demonstrated a main effect of stimulus or stimulus $\mathrm{x}$ trial interaction. A voxel-wise threshold of $p<$ 0.005 and a cluster volume larger than $225 \mathrm{~mm}^{3}$ (4 voxels of $3.75 \times 3.75 \times 4.00 \mathrm{~mm}$ dimension) was employed, resulting in a FWE corrected significance threshold of $p<0.05$. Prior work has demonstrated a relationship between the magnitude of the fMRI signal response within the amygdala and SCR production during Pavlovian fear conditioning [10, 17, 22, 39-41]. Therefore an anatomical mask was employed to include the amygdala in the group level regression analysis as well.

Prior work suggests that the amygdala and PFC produce and regulate the emotional response to aversive stimuli [19, 38]. Therefore, we completed an additional voxel-wise multiple regression analysis to identify anticipatory PFC, cingulate, and amygdala activity that varied with the threatrelated fMRI signal response. This analysis was limited to the functional ROI revealed by our repeated-measures ANOVA. Given that no CS was presented on UCS alone trials to elicit an anticipatory response, this analysis was restricted to $\mathrm{CS}+\mathrm{UCS}$ and $\mathrm{CS}-\mathrm{UCS}$ trials. This analysis included regressors for trial type (CS+UCS \& CS-UCS), threat-elicited fMRI signal response amplitude, and interaction of trial type and threat-elicited fMRI signal response amplitude. Monte Carlo simulations conducted in AFNI indicated that a voxel-wise threshold of $p<0.005$ and a cluster volume larger than $510 \mathrm{~mm}^{3}$ (9 voxels of $3.75 \mathrm{x}$ $3.75 \times 4.00 \mathrm{~mm}$ dimension) resulted in a FWE corrected significance threshold of $p<0.05$. Given our a priori hypotheses and the relatively small volume of the amygdala, we applied a small volume correction using a voxel-wise threshold of $p<0.005$ and a cluster volume larger than 112 $\mathrm{mm}^{3}$ ( 2 voxels of $3.75 \times 3.75 \times 4.00 \mathrm{~mm}$ dimension) for this area $(p<0.05$ corrected $)$.

\section{RESULTS}

UCS expectancy: Repeated-measures ANOVA revealed significant differences in UCS expectancy during the test trials. The results indicated there was a main effect for stimulus type $(F[1,20]=38.30, p<0.05)$ and a main effect for trial $(F[1,20]=33.68, p<0.05)$. There was no stimulus by trial interaction $(F<1.00)$. UCS expectancy was greater during Early test trials on CS+UCS [mean \pm SEM (adjusted for between subject variance [52]): $65.85 \pm 2.86 ; t[20]=$ $5.55, p<0.05]$ and CS-UCS $(70.60 \pm 3.97 ; t[20]=5.48, p<$ $0.05)$ than on UCS alone trials $(34.31 \pm 3.89)$. There was no difference in UCS expectancy for CS+UCS and CS-UCS $(t[20]=-1.00)$ presentations during Early test trials. UCS expectancy was greater during Late test trials on CS+UCS $(86.09 \pm 2.84 ; t[20]=6.20, p<0.05)$ and CS-UCS $(91.83 \pm$ $2.97 ; t[20]=6.29, p<0.05)$ compared to UCS alone $(48.58$ $\pm 4.56)$ trials. During the Late test trials, UCS expectancy was also greater during CS-UCS presentations than on $\mathrm{CS}+\mathrm{UCS}$ presentations $(t[20]=-2.31, p<0.05)$ (Fig. 2a).

Skin conductance response: Repeated-measures ANOVA also revealed significant differences in unconditioned SCR 


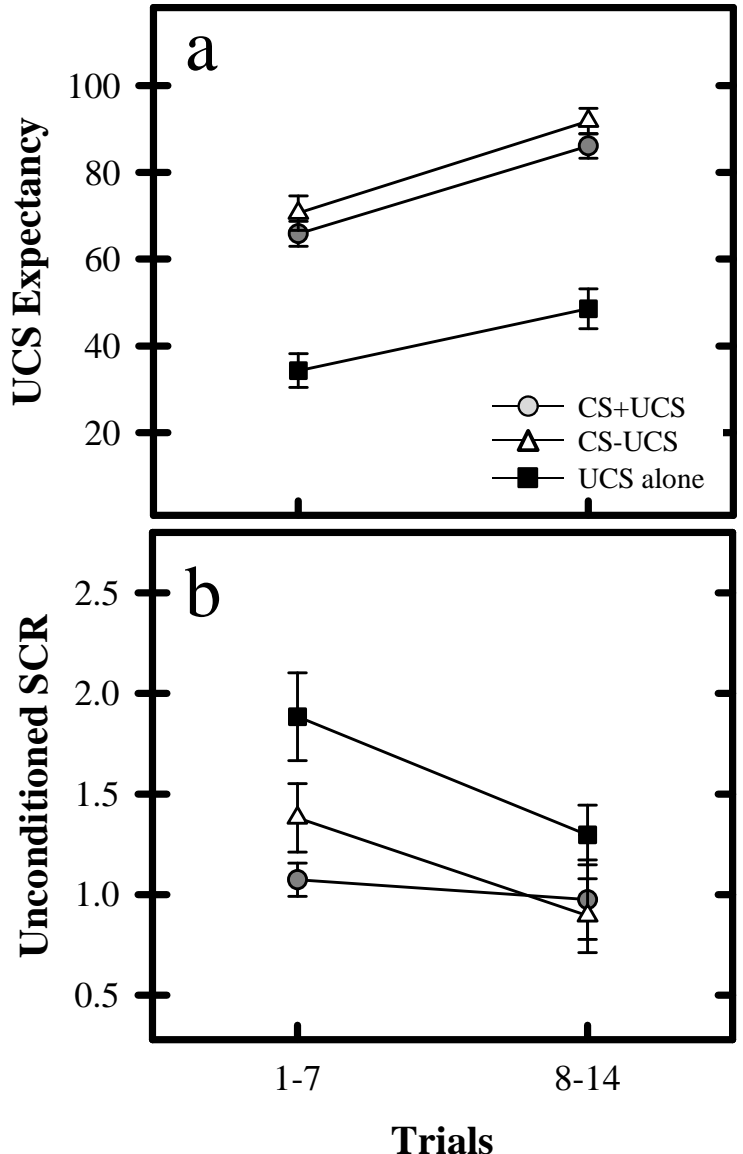

Fig. (2). UCS expectancy and unconditioned SCR. a) Learningrelated differences in UCS expectancy. UCS expectancy on Early and Late test trials was higher during CS+UCS and CS-UCS trials vs. UCS alone trials. b) Learning-related changes in unconditioned SCR expression were also observed. During Early test trials unconditioned SCRs were diminished on CS+UCS and CS-UCS trials compared to UCS alone trials. No differences were observed between CS+UCS and CS-UCS trials. During Late test trials no differences were observed between $\mathrm{CS}+\mathrm{UCS}$, CS-UCS, or UCS alone trials.

expression during the test trials. There was a main effect for stimulus type $(F[1,16]=4.85, p<0.05)$. There was also a trend for a main effect of trial $(F[1,16]=4.44, p=0.051)$, and a trend for a stimulus by trial interaction $(F[1,16]=4.39$, $p=0.052)$. Follow-up $t$-test comparisons revealed a diminished threat-elicited SCR for CS+UCS $(1.07 \pm 0.08$; $t[16]=-3.05, p<0.05)$ and CS-UCS trials $(1.38 \pm 0.17$; $t[16]=-2.04, p<0.05)$ compared to the UCS alone $(1.88 \pm$ 0.22 ) during Early test trials. There were no significant differences in unconditioned SCR between the CS+UCS and CS-UCS trials $(t[16]=-1.49)$ during Early test trials. Also, there were no differences in unconditioned SCR between the CS+UCS trials $(0.98 \pm 0.20)$ and CS-UCS trials $(0.90 \pm$ $0.18 ; t<1.00)$ during Late test trials. Unconditioned SCR during CS+UCS $(t[16]=-1.08)$ and CS-UCS $(t[16]=-1.38)$ trials also did not differ from UCS alone trials $(1.30 \pm 0.15)$ during the Late test trials (Fig. 2b).

Functional MRI: Repeated-measures ANOVA revealed significant differences in the magnitude of the threat-elicited
fMRI signal response within several brain regions (Tables 1 and 2; Fig. 3). Conditioned UCR diminution was observed within the dlPFC, dmPFC, vmPFC, and anterior insula replicating prior work $[10,15,17]$ (Table $\mathbf{1}$; Fig. 3). We also observed potentiation of the UCR within several brain regions including the dIPFC, IPL, and posterior insula (Table 2). Within each of these brain regions the threat-related fMRI signal response demonstrated a main effect for stimulus type $(F[20]>6.06 ; p<0.05$ corrected). A main effect for trial (i.e. Early vs. Late) was observed within the dIPFC, IPL, and anterior insula (Table 3). A stimulus $x$ trial interaction was observed within the left IPL (Table 2). $T$-test comparisons were conducted on the mean fMRI signal response from each area of activation that passed the significance threshold $(p<0.05$ corrected) for the main effect of stimulus type. There was no difference in the threatrelated fMRI signal response for CS+UCS compared to CS-UCS trials within the functional ROIs identified by the ANOVA. The unconditioned fMRI signal response was diminished for the CS+UCS and CS-UCS compared to the UCS alone within left dIPFC, dmPFC, vmPFC, and left anterior insula (Table 1). However, greater activation was observed for CS+UCS and CS-UCS trials compared to UCS alone trials within left dIPFC and bilateral IPL. Additionally, greater activation for CS-UCS vs. UCS alone trials was observed within bilateral posterior insula (Table 2).

A correlation analysis was performed on the mean percent signal change from the functional ROIs revealed by the ANOVA and our behavioral measures (i.e. trait anxiety level, UCS expectancy, and unconditioned SCR expression) (Tables 1 and 2; $p<0.05$ Bonferroni corrected). The threatrelated percent signal change within the functional ROI did not vary with trait anxiety or unconditioned SCR. These findings replicate prior work that used CS presentations that were easy to discriminate during Pavlovian fear conditioning [17]. However, a significant correlation was observed between UCS expectancy and the threat-elicited fMRI signal response within the dmPFC, vmPFC, left IPL, and left anterior insula (Tables $\mathbf{1}$ and 2), generally consistent with prior research [17]. A voxel-wise multiple linear regression analysis was conducted to evaluate brain activity within the functional ROI that varied with individual differences in behavior. The amygdala was also included in this analysis because prior work suggests this brain region mediates learning-related changes in SCR production [22,39-41]. The regression model accounted for stimulus type, trait anxiety, UCS expectancy, and unconditioned SCR. There were no brain regions within the functional ROI that varied with behavior (i.e. trait anxiety, UCS expectancy, or unconditioned SCR amplitude).

We also conducted a group level regression analysis to investigate whether anticipatory activation (i.e. the CR) within the PFC, cingulate, and amygdala varied with threatrelated activity (i.e. the UCR) from brain regions that demonstrated conditioned UCR diminution (Table 1). Anticipatory activity within dlPFC, dmPFC, vmPFC, ventrolateral PFC (vlPFC), posterior cingulate, and amygdala revealed a negative relationship with the threatelicited fMRI signal response (i.e. UCR) within many of the brain regions in which conditioned UCR diminution was observed (Table 4). Anticipatory activation within left vlPFC 
Table 1. Regions that Showed Conditioned Diminution of the UCR

\begin{tabular}{|c|c|c|c|c|c|c|c|c|c|c|}
\hline \multirow[b]{2}{*}{ Region } & \multirow[b]{2}{*}{$\operatorname{Vol}\left(\mathrm{mm}^{3}\right)$} & \multicolumn{3}{|c|}{ Talairach Coordinates } & \multirow{2}{*}{$\begin{array}{c}\text { CS+UCS vs. } \\
\text { CS-UCS } \\
t\end{array}$} & \multirow{2}{*}{$\begin{array}{c}\text { CS-UCS vs. } \\
\text { UCS Alone } \\
t\end{array}$} & \multirow{2}{*}{$\begin{array}{c}\text { CS+UCS vs. } \\
\text { UCS Alone } \\
t\end{array}$} & \multirow{2}{*}{$\begin{array}{c}\text { Trait } \\
r\end{array}$} & \multirow{2}{*}{$\begin{array}{c}\text { SCR } \\
r\end{array}$} & \multirow{2}{*}{$\begin{array}{c}\text { UCS } \\
\text { Expectancy }\end{array}$} \\
\hline & & $\mathbf{x}$ & $\mathbf{y}$ & $\mathbf{z}$ & & & & & & \\
\hline \multicolumn{11}{|l|}{ Dorsolateral PFC } \\
\hline Left & 3739 & -46.3 & 10.8 & 35.0 & n.s. & -4.23 & -3.81 & 0.04 & -0.04 & -0.17 \\
\hline Dorsomedial PFC & 5670 & -1.6 & 24.5 & 45.9 & n.s. & -4.52 & -4.18 & 0.04 & -0.04 & $-0.36^{*}$ \\
\hline Ventromedial PFC & 515 & -0.9 & 52.7 & -0.5 & n.s. & -4.33 & -3.96 & -0.02 & 0.05 & $-0.50^{*}$ \\
\hline \multicolumn{11}{|l|}{ Anterior Insula } \\
\hline Left & 1046 & -34.6 & 16.3 & 1.7 & n.s. & -3.73 & -3.99 & 0.06 & 0.18 & $-0.48^{*}$ \\
\hline
\end{tabular}

Note. Location, volumes, and coordinates from Talairach and Tournoux (1988) for the center of mass for areas of activation. Significance criteria: ANOVA $F[20]>6.06, p<0.05$ (corrected); $t[20] p<0.05$ (corrected). Significance criteria for two-tailed correlations: * indicates $p<0.05$ (corrected).
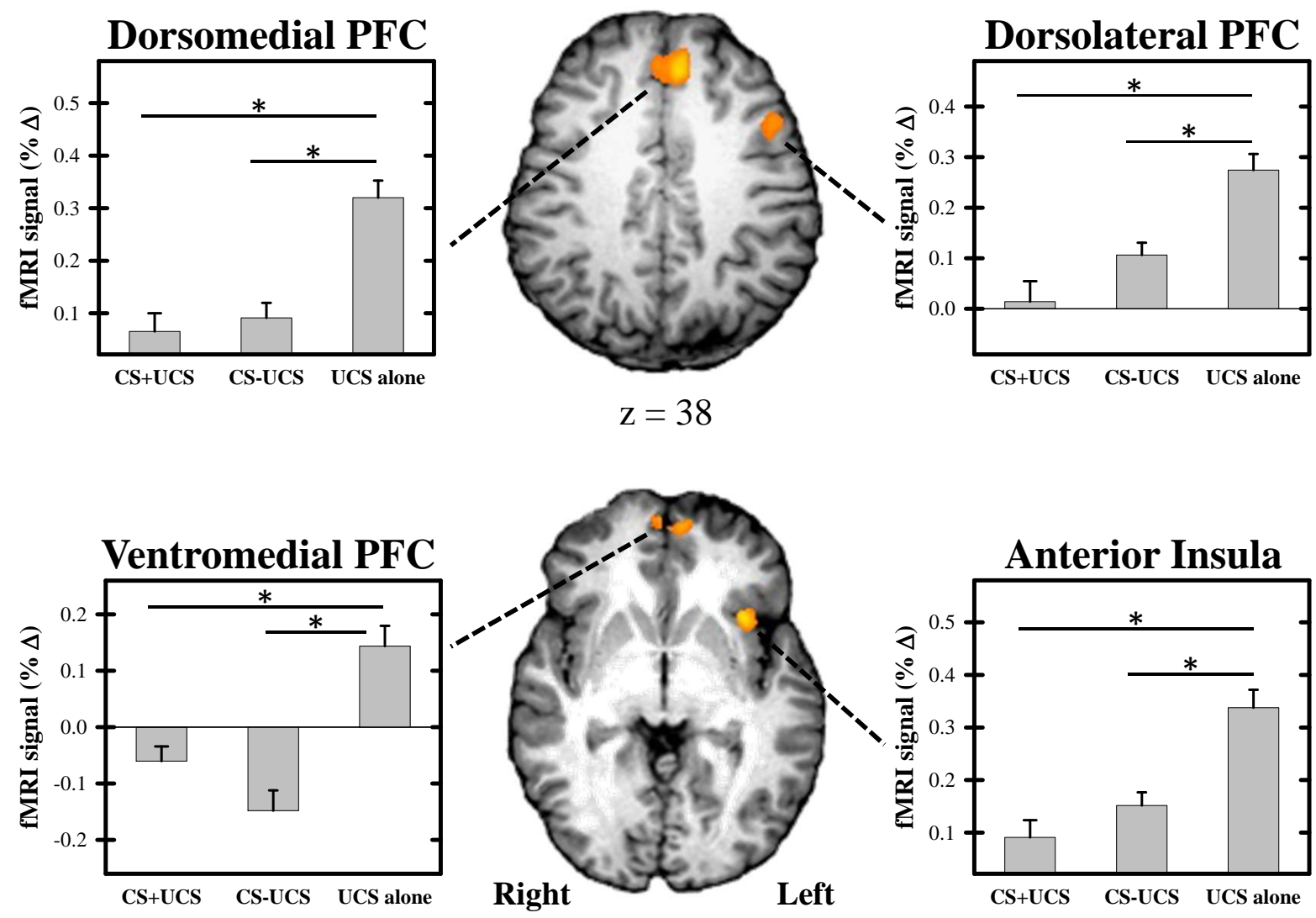

Fig. (3). UCR diminution within the fMRI signal response. Significant diminution of the unconditioned fMRI signal response was observed within the prefrontal cortex (PFC) and anterior insula during test trials. UCR amplitude within these brain regions was reduced when the UCS followed the CS+ (i.e. CS+UCS trials) and CS- (i.e. CS-UCS trials) compared to when the UCS was presented alone. There was no difference on CS+UCS versus CS-UCS trials. Graphs reflect the mean amplitude (\% signal change) of all voxels within volumes of activation. Error bars reflect SEM after adjusting for between-subject variance (Loftus and Masson, 1994). Asterisk indicates significant difference. 
Table 2. Regions that Showed Potentiation of the UCR

\begin{tabular}{|c|c|c|c|c|c|c|c|c|c|c|}
\hline \multirow[b]{2}{*}{ Region } & \multirow[b]{2}{*}{$\operatorname{Vol}\left(\mathrm{mm}^{3}\right)$} & \multicolumn{3}{|c|}{ Talairach Coordinates } & \multirow{2}{*}{$\begin{array}{c}\text { CS+UCS vs. } \\
\text { CS-UCS } \\
t\end{array}$} & \multirow{2}{*}{$\begin{array}{c}\text { CS-UCS vs. } \\
\text { UCS Alone } \\
t\end{array}$} & \multirow{2}{*}{$\begin{array}{c}\text { CS+UCS vs. } \\
\text { UCS Alone } \\
t\end{array}$} & \multirow{2}{*}{$\begin{array}{c}\text { Trait } \\
r\end{array}$} & \multirow{2}{*}{$\begin{array}{c}\text { SCR } \\
r\end{array}$} & \multirow{2}{*}{$\begin{array}{c}\begin{array}{c}\text { UCS } \\
\text { Expectancy }\end{array} \\
r\end{array}$} \\
\hline & & $\mathbf{x}$ & $\mathbf{y}$ & $\mathbf{z}$ & & & & & & \\
\hline \multicolumn{11}{|c|}{ Main effect of stimulus type } \\
\hline \multicolumn{11}{|l|}{ Dorsolateral PFC } \\
\hline \multicolumn{11}{|l|}{ Inf. Parietal Lobule } \\
\hline Right & 954 & 56.3 & -29.3 & 28.4 & n.s. & 3.82 & 3.45 & 0.32 & -0.06 & 0.28 \\
\hline Left & 2618 & -39.2 & -40.9 & 51.0 & n.s. & 3.85 & 3.44 & 0.15 & -0.07 & $0.43^{*}$ \\
\hline Left & 1054 & -60.9 & -27.0 & 27.7 & n.s. & 4.20 & 3.39 & 0.28 & -0.01 & 0.34 \\
\hline Left & 2106 & -41.9 & -14.2 & 12.6 & n.s. & 4.29 & n.s. & 0.22 & -0.12 & 0.31 \\
\hline \multicolumn{11}{|c|}{ Stimulus $x$ trial interaction } \\
\hline Inf. Parietal Lobule & 577 & -42.8 & -34.4 & 38.7 & & & & & & \\
\hline Early test trials & & & & & n.s. & n.s. & 4.11 & 0.14 & -0.06 & 0.33 \\
\hline Late test trials & & & & & n.s. & n.s. & n.s. & 0.05 & 0.09 & 0.20 \\
\hline
\end{tabular}

Note. Location, volumes, and coordinates from Talairach and Tournoux (1988) for the center of mass for areas of activation. Significance criteria: ANOVA $F[20]>6.06, p<0.05$ (corrected); $t[20] p<0.05$ (corrected). Significance criteria for two-tailed correlations: * indicates $p<0.05$ (corrected).

Table 3. Regions that Showed a Change Over Time

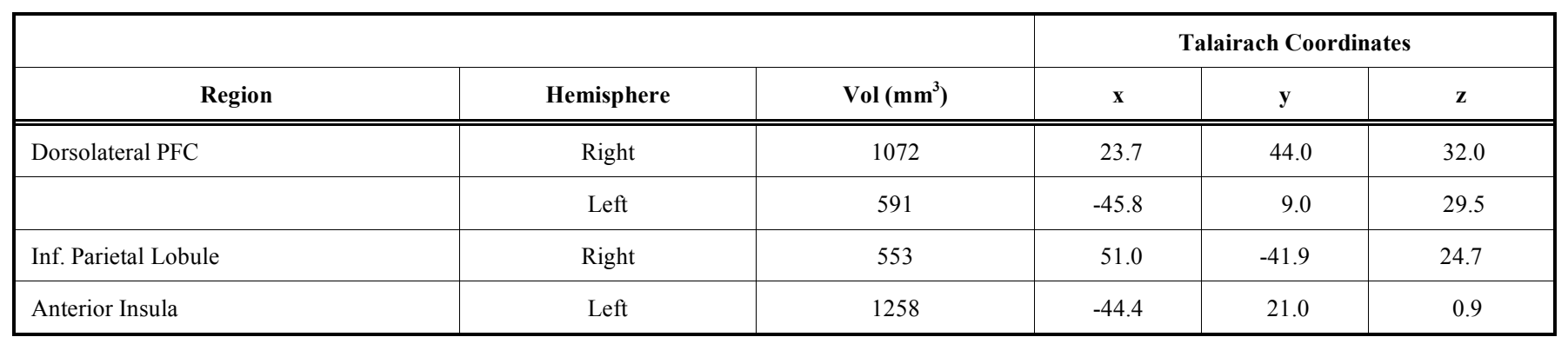

Note. Location, volumes, and coordinates from Talairach and Tournoux (1988) for the center of mass for areas of activation. Significance criteria: $F[20]>10.00 ; p<0.05$ (corrected).

Table 4. Regions Showing a Relationship between Anticipatory and Threat-Related Activity

\begin{tabular}{|c|c|c|c|c|c|c|c|c|c|c|}
\hline \multicolumn{5}{|c|}{ UCR Diminution Functional ROIs } & & \multicolumn{5}{|c|}{ Anticipatory Brain Activation } \\
\hline Region & $\frac{\text { Vol }}{\left(\mathbf{m m}^{3}\right)}$ & $\mathbf{x}$ & $\mathbf{y}$ & $\mathbf{z}$ & & Region & $\frac{\text { Vol }}{\left(\mathrm{mm}^{3}\right)}$ & $\mathbf{x}$ & $\mathbf{y}$ & $\mathbf{z}$ \\
\hline Dorsolateral PFC & & & & & $\leftrightarrow$ & Ventrolateral PFC & & & & \\
\hline Left & 3739 & -46.3 & 10.8 & 35.0 & & Left & 817 & -37.4 & 29.9 & -10.4 \\
\hline
\end{tabular}


Table 4. contd...

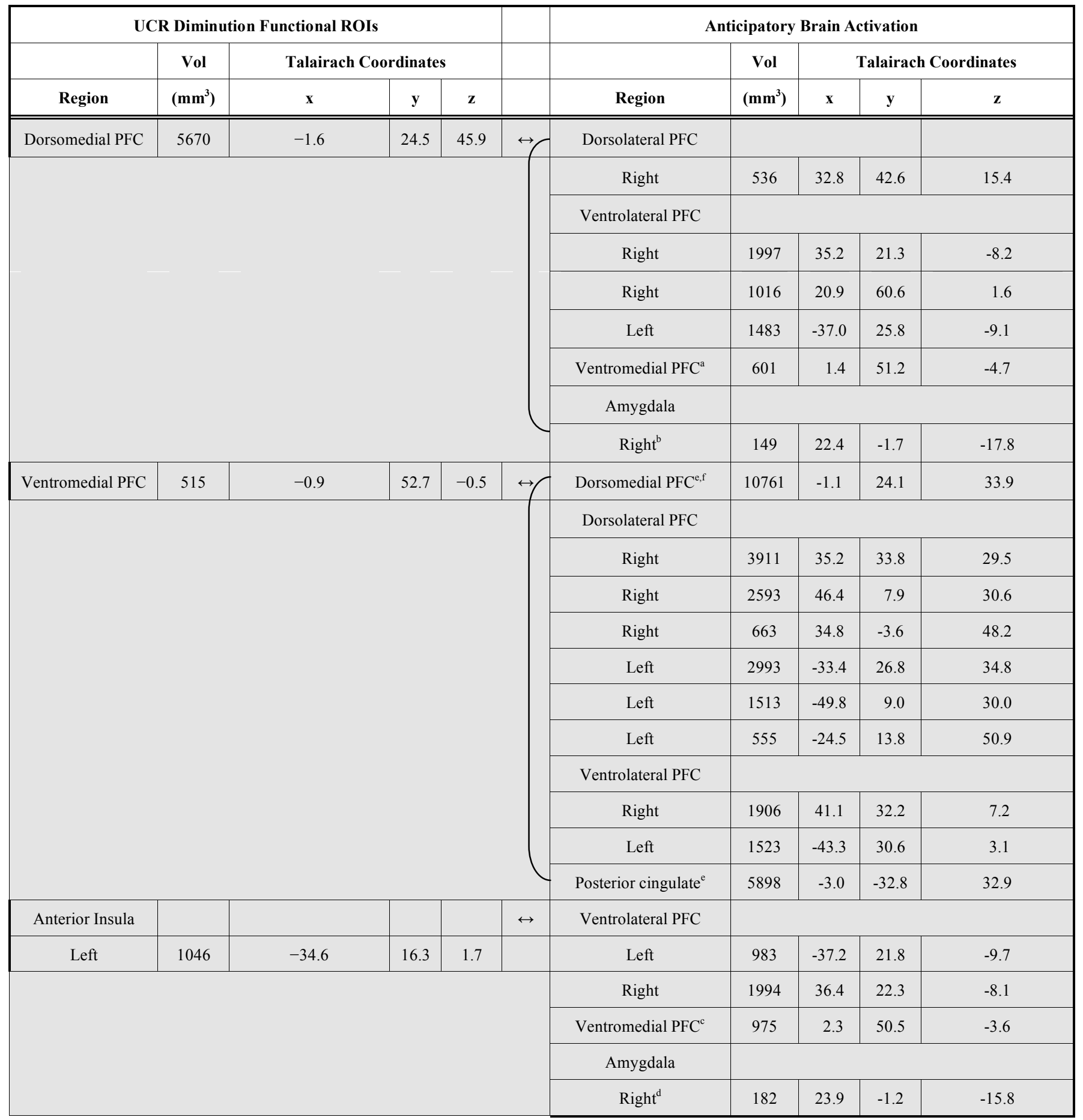

Note. Location, volumes, and coordinates from Talairach and Tournoux (1988) for the center of mass for areas of activation. Significance criteria: $\mathrm{t}[20]>2.98, p<0.05$ (corrected). The UCR amplitude (from ROI on left side of table) varied with the anticipatory response (i.e. the CR) within the dorsolateral, dorsomedial PFC, ventromedial PFC, and posterior cingulate (right side of the table). Exploratory analysis of amygdala and hippocampal activation small volume correction applied t[20] $>2.98,112 \mathrm{~mm} 3, p<0.05$ (corrected) (right side of the table). Letters (a-f) correspond to images and graphical representation presented in Fig. (4).

was negatively correlated with the threat response within each of the brain areas that showed UCR diminution. This effect was also observed between anticipatory activation within the right vIPFC and threat-related activity within each of the functional ROI that showed UCR diminution, except for the left dlPFC (Table 4). A negative relationship was also observed between anticipatory activity within vmPFC and the threat-related response within left $\mathrm{dlPFC}, \mathrm{dmPFC}$, and left anterior insula (Fig. 4a and c). A similar pattern was observed between anticipatory activation within dlPFC, $\mathrm{dmPFC}$, vlPFC, and $\mathrm{PCC}$ and the threat-related response within vmPFC (Fig. 4e). Finally, anticipatory amygdala activity showed a similar negative relationship with the threat-related response within $\mathrm{dmPFC}$ and left anterior insula (Fig. $4 \mathbf{b}$ and d). 


\section{Relationship between Anticipatory and Threat-related Activity}

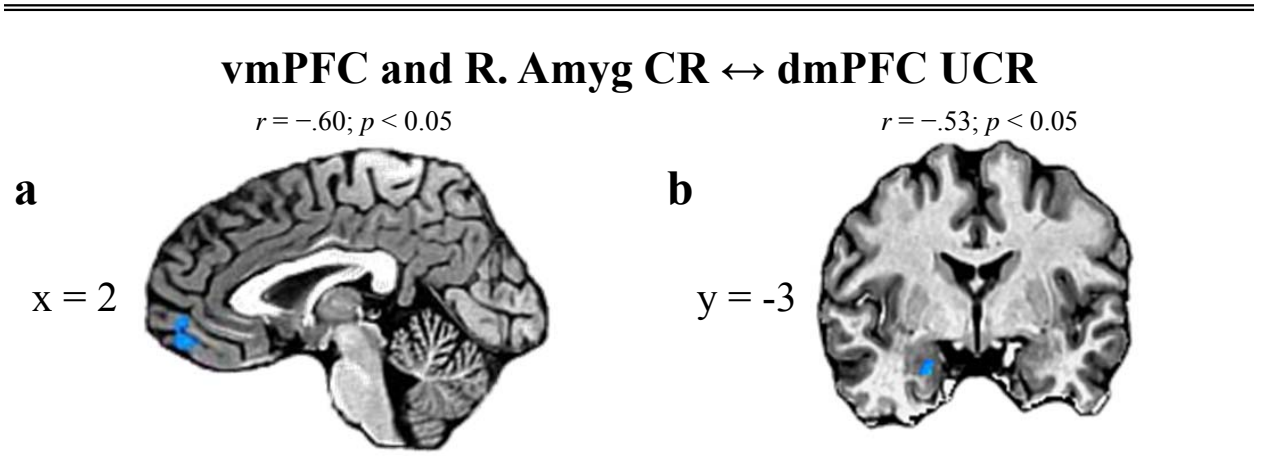

vmPFC and R. Amyg CR Ant. Insula UCR
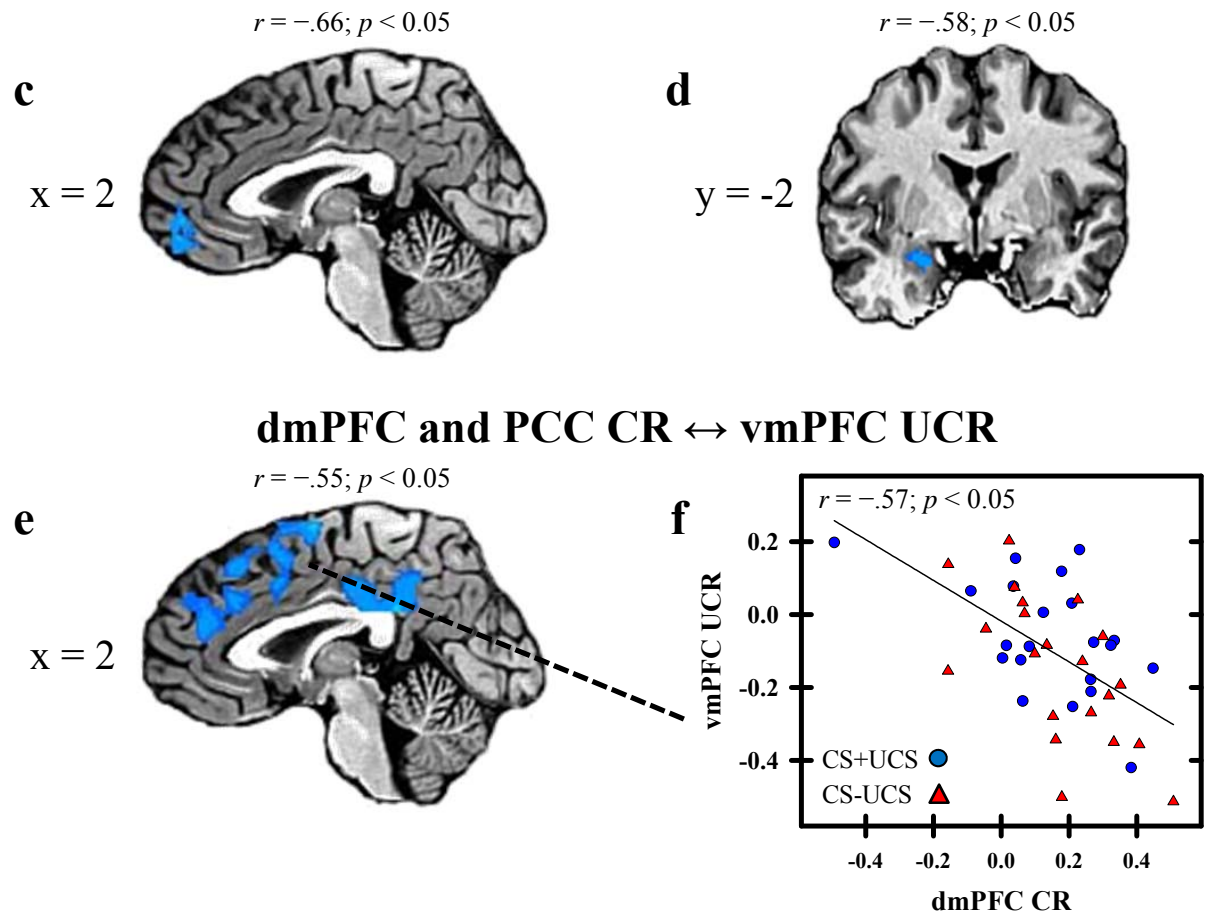

Fig. (4). Relationship between anticipatory and threat-related activity. Threat-related activity, extracted from the ROI depicted in Table 1 and Figure 3, was included in a regression analysis to investigate differences in the relationship between anticipatory activity (i.e. CR) and threatrelated activity (i.e. UCR) on CS+UCS and CS-UCS trials. A negative relationship between anticipatory and threat-related activity (\% signal change) was observed in several areas of the PFC, cingulate, and amygdala (a-e). Correlation values comparing the anticipatory and threatrelated response within these brain areas are presented above the brain images. The correlation value above image (e) represents activation observed between PCC CR and vmPFC UCR. The correlation value for dmPFC CR and vmPFC UCR is presented in graph (f). Talairach coordinates for the depicted areas of activation are presented in Table $\mathbf{4}$ and labeled with letters (a-e) corresponding to each image above.

\section{DISCUSSION}

The ability to learn cues that predict threats is critical to survival. These cue-threat relationships are established when outcomes violate our expectations [53]. These relationships are supported by associative learning and expectancy-related processes that may somewhat independently influence the magnitude of the threat-elicited response [10-12, 15, 17]. Therefore, the present study used CS presentations that were difficult to discriminate to investigate the neural processes that support conditioned UCR diminution in the absence of differential UCS expectancies to better understand associative learning processes that mediate UCR diminution.

In the present study, we observed learning-related changes in UCS expectancy and unconditioned SCR expression. By design, UCS expectancy was high on CS+UCS and CS-UCS trials. In contrast, UCS expectancy on UCS alone trials was rated around 50 (Fig. 2a). These findings demonstrate that volunteers expected the UCS following the $\mathrm{CS}+$ and $\mathrm{CS}^{-}$, but remained uncertain about the timing of the UCS alone during the conditioning session. 
UCS expectancy was also associated with the amplitude of threat-elicited SCR expression. Specifically, unconditioned SCR amplitude was decreased on CS+UCS and CS-UCS trials (when UCS expectancy was high) compared to the UCS alone (when UCS expectancy was lower) on Early test trials (Fig. 2b). These findings are consistent with prior work that has shown a decreased UCR when the UCS is predictable vs. unpredictable $[8,9,11,54]$. Based on our pilot data, we also expected to observe greater unconditioned SCR diminution during CS+UCS than CS-UCS trials in this study. Findings of differential SCR, with equivalent UCS expectancy, would allow us to address questions related to learning independent of conscious expectations. However, no difference in unconditioned SCR expression was observed between CS+UCS and CS-UCS trials. Therefore, there is no evidence of conditioned UCR diminution that is independent of UCS expectancy in the present study. The lack of differential SCRs on CS+UCS vs. CS-UCS trials is likely due to the lack of discriminative control gained by the $\mathrm{CS}$. Given that the CS+ and $\mathrm{CS}-$ were difficult to discriminate, evidenced by high expectancy ratings, participants appear to have interpreted the acquisition phase as a $50 \%$ reinforcement schedule of a single CS rather than separate presentations of a CS+ and CS-. Therefore, the lack of differential unconditioned SCRs during CS+UCS compared to CS-UCS trials appears to be due to a deficit in learning the CS discrimination.

Although there is no evidence of learning, independent of expectancy, we did observe conditioned UCR diminution in other contrasts in the present study. Unconditioned SCR amplitude was diminished on CS+UCS and CS-UCS trials compared to the UCS alone trials. This pattern was also observed in the fMRI signal within the left dIPFC $(z=35.0)$, $\mathrm{dmPFC}, \mathrm{vmPFC}$, and left anterior insula. These findings are generally consistent with prior studies that have shown UCR diminution within these brain regions [10, 15, 17]. However, contrary to our prior work, an enhanced threat-related fMRI response was observed within other brain regions including a more superior region of left dIPFC $(\mathrm{z}=51.7)$, bilateral IPL, and bilateral posterior insula. Similar to the SCR data, there were no differences between CS+UCS and CS-UCS trials within any of the brain regions that demonstrated learningrelated changes in the threat-elicited fMRI signal response (Tables 1 and 2). Taken together, the present findings replicate prior studies that have shown learning-related changes in brain activation that resemble the pattern of the emotional response produced [15, 17, 22, 39, 40].

Prior work suggests that UCS expectancy modulates the magnitude of the threat-related fMRI signal response [10, 15, 17]. Prior work has also shown that conscious expectation influences the emotional response produced by a threat $[10$, 11, 15-17]. Similar results were observed in the current study. Consistent with our prior work, a negative relationship was observed between UCS expectancy and brain activity in regions that showed conditioned UCR diminution [17]. Specifically, a negative relationship was observed between UCS expectancy and brain activation within dmPFC, vmPFC, and left anterior insula (Table 1). However, a positive relationship was observed between UCS expectancy and the amplitude of the threat-related fMRI signal response within left IPL (Table 2). This relationship between the unconditioned fMRI signal response and UCS expectancy was not observed in our group level multiple linear regression analysis. The regression analysis was conducted on a voxel-wise basis and accounted for additional measures of interest (e.g. stimulus type). These findings suggest that UCS expectancy varied with the mean percent signal change of the threat-related fMRI signal response within the ROIs as a whole. However, UCS expectancy did not explain unique variance within the threat-related fMRI signal response within these brain regions. These findings replicate prior conditioning work that employed CS presentations that were easy to discriminate [17]. In addition, these data support previous research that suggests that regions of the PFC support associative learning processes [55, 56].

The PFC appears to support top-down processes that are important for emotion regulation. Prior work has demonstrated a negative relationship between anticipatory PFC activity and the threat-related response within the amygdala $[17,38]$. Specifically, as anticipatory PFC activity increased, the neural response to the threat decreased [17]. The data from the current study are generally consistent with this previous research. In the current study, a negative relationship was observed between anticipatory activation within the PFC and threat-related activity within brain regions that showed UCR diminution (Table 1). Specifically, as anticipatory activity within dIPFC, dmPFC, vlPFC, vmPFC, and the amygdala increased the threat-related response within left dIPFC, dmPFC, vmPFC, and anterior insula decreased (Table 4, Fig. 4). These findings demonstrate that anticipatory brain activity affects the response elicited by a threat. The primary focus of fear conditioning research has typically been centered on the anticipatory response to a threat. This prior work has contributed to the development of cognitive, behavioral, and drug interventions [28, 57-59] and has provided a better understanding of the aberrant top-down emotion regulation processes that are observed in conditions such as posttraumatic stress disorder (PTSD) [27, 29, 60-62]. The current findings demonstrate neural mechanisms that influence the threat-related emotional response and may offer novel insights for future clinical research on emotion regulation.

In summary, conditioned diminution of the unconditioned SCR and fMRI signal response was observed during Pavlovian fear conditioning. UCR diminution was observed within left dlPFC, dmPFC, vmPFC, and left anterior insula. Consistent with prior work, many of the brain regions that showed learning-related changes in the threat-related fMRI signal response varied with UCS expectancy [10, 15, 17]. Further, a negative relationship was observed between the brain regions that showed UCR diminution and anticipatory PFC activation (i.e. dlPFC, dmPFC, vlPFC, and vmPFC). This finding supports prior work that suggests top-down mechanisms provided by the PFC inhibit the emotional response produced by a threat. The findings from the current study provide a better understanding of the neural mechanisms that support associative learning processes that modulate the response to a threat.

\section{ACKNOWLEDGEMENTS}

This research was supported by the University of Alabama at Birmingham Faculty Development Grant Program and NIH R01 MH098348 (DCK). 


\section{ABBREVIATIONS}

$\begin{array}{ll}\mathrm{CS} & =\text { conditioned stimulus, } \\ \mathrm{UCS} & =\begin{array}{l}\text { } \\ \mathrm{CS}+\end{array} \\ \mathrm{CS}+\mathrm{UCS} \text { paired with } & =\mathrm{CS}+\text { paired with the UCS on test trials, } \\ \mathrm{CS}- & =\mathrm{CS} \text { presented alone, } \\ \mathrm{CS}-\mathrm{UCS} & =\mathrm{CS}-\text { paired with the UCS on test trials, } \\ \mathrm{CR} & =\text { conditioned response, } \\ \mathrm{UCR} & =\text { unconditioned response, } \\ \mathrm{SCR} & =\text { skin conductance response, } \\ \mathrm{PFC} & =\text { prefrontal cortex, } \\ \mathrm{IPL} & =\text { inferior parietal lobule }\end{array}$

\section{REFERENCES}

[1] Kim JJ, Jung MW. Neural circuits and mechanisms involved in Pavlovian fear conditioning: a critical review. Neurosci Biobehav Rev 2006; 30(2): 188-202.

[2] LeDoux J. The amygdala. Curr Biol CB 2007; 17(20): 868-74.

[3] Mineka S, Öhman A. Born to fear: non-associative vs associative factors in the etiology of phobias. Behav Res Ther 2002; 40(2): 173-84.

[4] Domjan M. Pavlovian conditioning: a functional perspective. Annu Rev Psychol 2005; 56: 179-206.

[5] Franchina JJ. Escape behavior and shock intensity: within subject versus between-groups comparisons. J Comp Physiol Psychol 1969; 69(2): 241-5.

[6] Helmstetter FJ, Bellgowan PS. Lesions of the amygdala block conditional hypoalgesia on the tail flick test. Brain Res 1993; 612(1-2): 253-7.

[7] Kamin LJ. Traumatic avoidance learning: the effects of CS-US interval with a trace-conditioning procedure. J Comp Physiol Psychol 1954; 47(1): 65-72.

[8] Baxter R. Diminution and recovery of the UCR in delayed and trace classical GSR conditioning. J Exp Psychol 1966; 71(3): 44751.

[9] Kimmel E. Judgments of UCS intensity and diminution of the UCR in classical GSR conditioning. J Exp Psychol 1967; 73: 532-43.

[10] Knight DC, Waters NS, King MK, Bandettini PA. Learning-related diminution of unconditioned SCR and fMRI signal responses. Neuroimage Elsevier B 2010; 49(1): 843-8

[11] Knight DC, Lewis EP, Wood KH. Conditioned diminution of the unconditioned skin conductance response. Behav Neurosci 2011; 125(4): 626-31.

[12] Marcos JL, Redondo J. Effects of conditioned stimulus presentation on diminution of the unconditioned response in aversive classical conditioning. Biol Psychol 1999; 50(2): 89-102.

[13] Lykken DT, Macindoe I, Tellegen A. Preception: autonomic response to shock as a function of predictability in time and locus. Psychophysiology 1972; 9(3): 318-33.

[14] Peeke SC, Grings WW. Magnitude of UCR as a function of variability in the CS-UCS relationship. J Exp Psychol 1968; 77: 16.

[15] Dunsmoor JE, Bandettini PA, Knight DC. Neural correlates of unconditioned response diminution during Pavlovian conditioning. Neuroimage 2008; 40(2): 811-7.

[16] Rust J. Unconditioned response diminution in the skin resistance response. J Gen Psychol 1976; 95: 77-84.

[17] Wood KH, Ver Hoef LW, Knight DC. Neural mechanisms underlying the conditioned diminution of the unconditioned fear response. Neuroimage Elsevier Inc 2012; 60(1): 787-99.

[18] Linnman C, Rougemont-Bücking A, Beucke JC, Zeffiro TA, Milad MR. Unconditioned responses and functional fear networks in human classical conditioning. Behav Brain Res Elsevier BV 2011; 221(1): $237-45$
[19] Delgado MR, Nearing KI, Ledoux JE, Phelps EA. Neural circuitry underlying the regulation of conditioned fear and its relation to extinction. Neuron 2008; 59(5): 829-38.

[20] Ochsner KN, Bunge SA, Gross JJ, Gabrieli JD. Rethinking feelings: an fMRI study of the cognitive regulation of emotion. J Cogn Neurosci 2002; 14: 1215-29.

[21] Milad MR, Vidal-Gonzalez I, Quirk GJ. Electrical stimulation of medial prefrontal cortex reduces conditioned fear in a temporally specific manner. Behav Neurosci 2004; 118(2): 389-94.

[22] Knight DC, Nguyen HT, Bandettini PA. The role of the human amygdala in the production of conditioned fear responses. Neuroimage 2005; 26(4): 1193-200.

[23] Basten U, Stelzel C, Fiebach CJ. Trait anxiety modulates the neural efficiency of inhibitory control. J Cogn Neurosci 2011; 10: 313245

[24] Klumpp H, Ho SS, Taylor SF, Phan KL, Abelson JL, Liberzon I Trait anxiety modulates anterior cingulate activation to threat interference. Depress Anxiety 2011; 28(3): 194-201

[25] Nitschke JB, Sarinopoulos I, Mackiewicz KL, Schaefer HS, Davidson RJ. Functional neuroanatomy of aversion and its anticipation. Neuroimage 2006; 29(1): 106-16.

[26] Etkin A, Wager TD. Functional neuroimaging of anxiety: a metaanalysis of emotional processing in ptsd, social anxiety disorder, and specific phobia. Am J Psychiatry 2007; 164: 1476-88.

[27] Milad MR, Rauch SL, Pitman RK, Quirk GJ. Fear extinction in rats: implications for human brain imaging and anxiety disorders. Biol Psychol 2006; 73(1): 61-71.

[28] Milad MR, Pitman RK, Ellis CB, et al. Neurobiological basis of failure to recall extinction memory in posttraumatic stress disorder Biol Psychiatry Elsevier Inc 2009; 66(12):1075-82.

[29] Rauch SL, Shin LM, Phelps EA. Neurocircuitry models of posttraumatic stress disorder and extinction: human neuroimaging research--past, present, and future. Biol Psychiatry 2006; 60(4): 376-82.

[30] Sehlmeyer C, Dannlowski U, Schöning S, et al. Neural correlates of trait anxiety in fear extinction. Psychol Med 2011; 41(4): 78998.

[31] Somerville LH, Kim H, Johnstone T, Alexander AL, Whalen PJ. Human amygdala responses during presentation of happy and neutral faces: Correlations with state anxiety. Biol Psychiatry 2004; 55(9): 897-903.

[32] Cook EW 3rd, Davis TL, Hawk LW, Spence EL, Gautier CH. Fearfulness and startle potentiation during aversive visual stimuli. Psychophysiology 1992; 29(6): 633-45.

[33] Grillon C, Ameli R, Goddard A, Woods SW, Davis M. Baseline and fear-potentiated startle in panic disorder patients. Biol Psychiatry 1994; 35: 431-9.

[34] Indovina I, Robbins TW, Núñez-Elizalde AO, Dunn BD, Bishop SJ. Fear-conditioning mechanisms associated with trait vulnerability to anxiety in humans. Neuron 2011; 69(3): 563-71.

[35] Casey BJ, Thomas KM, Welsh TF, et al. Dissociation of response conflict, attentional selection, and expectancy with functional magnetic resonance imaging. Proc Natl Acad Sci USA 2000; 97(15): 8728-33.

[36] Fletcher PC, Anderson JM, Shanks DR, et al. Responses of human frontal cortex to surprising events are predicted by formal associative learning theory. Nat Neurosci 2001; 4(10): 1043-8.

[37] Dunsmoor JE, Bandettini PA, Knight DC. Impact of continuous versus intermittent CS-UCS pairing on human brain activation during Pavlovian fear conditioning. Behav Neurosci 2007; 121(4): 635-42.

[38] Sarinopoulos I, Grupe DW, Mackiewicz KL, et al. Uncertainty during anticipation modulates neural responses to aversion in human insula and amygdala. Cereb Cortex 2010; 20(4): 929-40.

[39] Cheng DT, Knight DC, Smith CN, Stein EA, Helmstetter FJ. Functional MRI of human amygdala activity during Pavlovian fear conditioning: stimulus processing versus response expression. Behav Neurosci 2003; 117(1): 3-10.

[40] Cheng DT, Knight DC, Smith CN, Helmstetter FJ. Human amygdala activity during the expression of fear responses. Behav Neurosci 2006; 120(6): 1187-95.

[41] Cheng DT, Richards J, Helmstetter FJ. Activity in the human amygdala corresponds to early, rather than late period autonomic responses to a signal for shock. Learn Mem 2007; 14(7): 485-90.

[42] Spielberger C. State-Trait Anxiety Inventory for Adults. Redwood City, CA: Mind Garden 1983 
[43] Grös DF, Antony MM, Simms LJ, McCabe RE. Psychometric properties of the state-trait inventory for cognitive and somatic anxiety (STICSA): comparison to the state-trait anxiety inventory (STAI). Psychol Assess 2007; 19(4): 369-81.

[44] Knight DC, Wood KH. Investigating the neural mechanisms of aware and unaware fear memory with FMRI. J Vis Exp JoVE 2011; 56: 1-6.

[45] Ogawa S, Lee TM, Kay AR, Tank DW. Brain magnetic resonance imaging with contrast dependent on blood oxygenation. Proc Natl Acad Sci USA 1990; 87(24):9868-72.

[46] Rick J, Speck O, Maier S, et al. Optimized EPI for fMRI using a slice-dependent template-based gradient compensation method to recover local susceptibility-induced signal loss. MAGMA 2010; 23(3): $165-76$

[47] Cox RW. AFNI: software for analysis and visualization of functional magnetic resonance neuroimages. Comput Biomed Res 1996; 29(3): 162-73.

[48] Talairach J, Tournoux P. Co-planar Stereotaxic Atlas of the Human Brain. New York: Thieme 1988

[49] Friston KJ, Ashburner J, Frith CD, Poline J, Heather JD, Frackowiak RSJ. Spatial registration and normalization of images. Hum Brain Mapp 1995; 2: 165-89.

[50] Forman S, Cohen J, Fitzgerald M, Eddy W, Mintun M, Noll D. Improved assessment of significant activation in functional magnetic resonance imaging (fMRI): use of a cluster-size threshold. Magn Reson Med 1995; 33(5): 636-47.

[51] Saad ZS, Chen G, Reynolds RC, et al. Functional imaging analysis contest (FIAC) analysis according to AFNI and SUMA. Hum Brain Mapp 2006; 27(5): 417-24.

[52] Loftus GR, Masson MEJ. Using confidence intervals in withinsubject designs. Psychon Bull Rev 1994; 1(4): 476-90.
[53] Rescorla RA. Behavioral studies of Pavlovian conditioning. Annu Rev Neurosci 1988; 11: 329-52.

[54] Orlebeke JF, van Doornen LJ. Preception (UCR diminution) in normal and neurotic subjects. Biol Psychol 1977; 5(1): 15-22.

[55] Knight DC, Smith CN, Cheng DT, Stein EA, Helmstetter FJ. Amygdala and hippocampal activity during acquisition and extinction of human fear conditioning. Cogn Affect Behav Neurosci 2004; 4(3): 317-25.

[56] Phillips ML, Drevets WC, Rauch SL, Lane R. Neurobiology of emotion perception I: the neural basis of normal emotion perception. Biol Psychiatry 2003; 54(5): 504-14.

[57] Jovanovic T, Ressler KJ. How the neurocircuitry and genetics of fear inhibition may inform our understanding of PTSD. Am J Psychiatry 2010; 167(6): 648-62.

[58] Ressler KJ, Rothbaum BO, Tannenbaum L, Anderson P. Cognitive enhancers as adjuncts to psychotherapy. Arch Gen Psychiatry 2004; 61

[59] Vervliet B, Vansteenwegen D, Eelen P. Generalization of extinguished skin conductance responding in human fear conditioning. Learn Mem 2004; 11(5): 555-8.

[60] King AP, Abelson JL, Britton JC, Phan KL, Taylor SF, Liberzon I. Medial prefrontal cortex and right insula activity predict plasma ACTH response to trauma recall. Neuroimage. Elsevier Inc 2009; 47(3): 872-80.

[61] Parsons RG, Ressler KJ. Implications of memory modulation for post-traumatic stress and fear disorders. Nat Neurosci 2013; 16(2): 146-53.

[62] Yehuda R, LeDoux J. Response variation following trauma: a translational neuroscience approach to understanding PTSD. Neuron 2007; 56(1): 19-32. 